\title{
Stabilization of a Virus-Like Particle and Its Application as a Nanoreactor at Physiological Conditions
}

\author{
Lise Schoonen, ${ }^{\ddagger}$ Sjors Maassen, ${ }^{\ddagger}$ Roeland J. M. Nolte, ${ }^{\ddagger}$ and Jan C. M. van Hest ${ }^{*}, \neq \ddagger$ \\ ${ }^{\dagger}$ Eindhoven University of Technology, P.O. Box 513 (STO 3.31), 5600 MB Eindhoven, The Netherlands \\ ${ }^{\ddagger}$ Radboud University, Institute for Molecules and Materials, Heyendaalseweg 135, 6525 AJ Nijmegen, The Netherlands
}

\section{Supporting Information}

ABSTRACT: Virus-like particles are very interesting tools for application in bionanotechnology, due to their monodisperse features and biocompatibility. In particular, the cowpea chlorotic mottle virus (CCMV) capsid has been studied extensively as it can be assembled and disassembled reversibly, facilitating cargo encapsulation. CCMV is, however, only stable at physiological conditions when its endogenous nucleic acid cargo is present. To gain more flexibility in the type of cargo encapsulated and to broaden the window of operation, it is interesting to improve the stability of the empty virus-like particles. Here, a method is described to utilize the CCMV capsid at close to physiological conditions as a stable, enzyme-

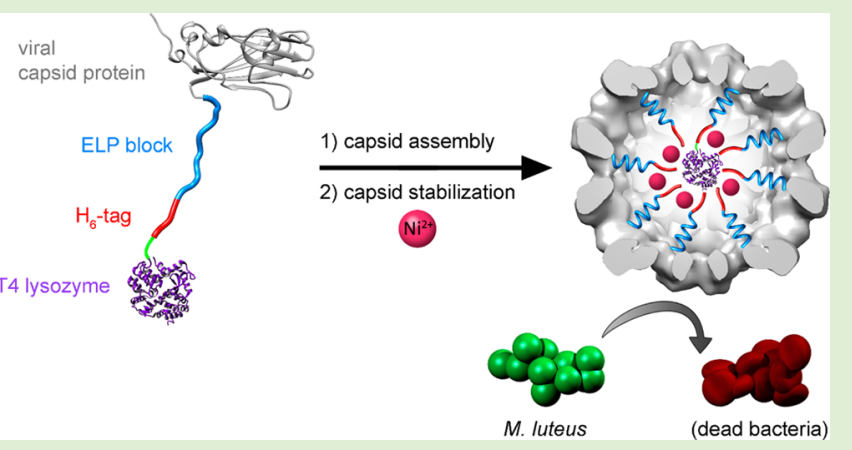
filled nanoreactor. As a proof-of-principle, the encapsulation of T4 lysozyme (T4L) was chosen; this enzyme is a promising antibiotic, but its clinical application is hampered by, for example, its cationic character. It was shown that four T4L molecules can successfully be encapsulated inside CCMV capsids, while remaining catalytically active, which could thus improve the enzyme's application potential.

\section{INTRODUCTION}

Virus-like particles (VLPs) are protein-based nanocages composed of a discrete number of viral capsid proteins. ${ }^{1}$ In contrast to some other types of nanoparticles, such as liposomes or polymeric vesicles, VLPs are very monodisperse in shape and size. Of special interest is the cowpea chlorotic mottle virus (CCMV), an icosahedral plant virus, which normally adopts $T=3$ symmetry. This capsid consists of 180 identical $20 \mathrm{kDa}$ capsid proteins and has an inner and outer diameter of 18 and $28 \mathrm{~nm}$, respectively. ${ }^{2,3}$ The CCMV VLP is able to undergo reversible, $\mathrm{pH}$-dependent assembly and disassembly, even in the absence of its viral RNA. ${ }^{4}$ At $\mathrm{pH}$ 7.5 , the capsid disassembles into 90 protein dimers, and by lowering the $\mathrm{pH}$ to 5.0 , the original structure is reformed. This property has been used to encapsulate inorganic materials, negatively charged polymers, ${ }^{7,8}$ and enzymes ${ }^{9}$ by adding these molecules to the media during the assembly process of the protein dimers.

The endogenous assembly behavior of CCMV VLPs does not allow for their facile application in biological settings, as the capsids will disassemble at a physiologically neutral $\mathrm{pH}$. Therefore, a modified protein-based block copolymer of the CCMV capsid protein, containing an $\mathrm{N}$-terminal elastin-like polypeptide (ELP) block, was previously developed in our lab. ${ }^{10}$ ELPs are stimulus-responsive polymers, derived from elastin, which can switch from a water-soluble state to a collapsed, insoluble state, upon a change in the environmental conditions, such as temperature, $\mathrm{pH}$, (bivalent) cations, and salt concentration of the solution. ${ }^{11,12}$ ELPs consist of repeats of the VPGXG pentapeptide, where $\mathrm{X}$ is any natural amino acid except proline. Previously, the stimulus-responsive polypeptide block was placed at the N-terminal end of the CCMV capsid protein, replacing the cationic RNA-binding domain. The introduction of the ELP resulted in a new assembly pathway: ELP-induced assembly resulted in the formation of $T=1$ capsids, consisting of 30 protein dimers, after an increase of the salt concentration at $\mathrm{pH}$ 7.5. Capsids with a $T=3$ symmetry could still be formed via the endogenous $\mathrm{pH}$-induced assembly pathway.

Even though the newly developed ELP-CCMV capsid proteins could assemble at physiological $\mathrm{pH}$, still a high salt concentration $(\sim 2 \mathrm{M} \mathrm{NaCl})$ was needed to form the capsids. Therefore, a third assembly mechanism for the ELP-CCMV capsid proteins was introduced, making use of the metalbinding properties of the hexahistidine tags at the $\mathrm{N}$-terminus of ELP-CCMV, which had initially been introduced to facilitate protein purification through affinity chromatography. ${ }^{13}$ The addition of divalent ions to ELP-CCMV, such as nickel or cobalt, resulted in local clustering of the capsid proteins,

Special Issue: Organized Peptidic Nanostructures as Functional Materials

Received: May 5, 2017

Revised: June 15, 2017

Published: June 20, 2017 


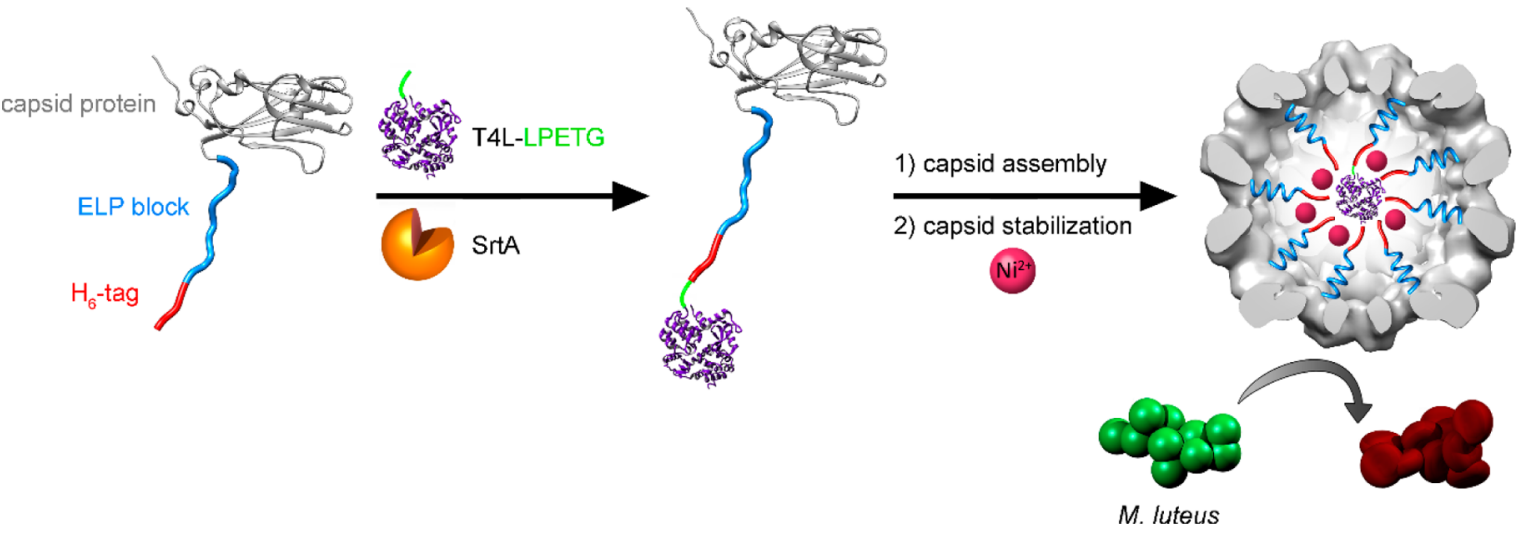

Figure 1. Schematic representation of the Sortase A-mediated N-terminal functionalization of hexahistidine-tagged ELP-CCMV capsid protein dimers with LPETG-modified T4 lysozyme and subsequent enzyme encapsulation via capsid formation. Capsid stabilization with $\mathrm{Ni}^{2+}$ ions results in capsids which are stable at low $\mathrm{NaCl}$ concentrations and allows the degradation of the cell wall of $M$. luteus by lysozyme. Adapted in part with permission from ref 13. Copyright 2016 Wiley-VCH Verlag GmbH \& Co. KGaA, Weinheim.

lowering the transition temperature of the ELP blocks and subsequently leading to capsid assembly at $\mathrm{pH} 7.5$, without the need for a high salt concentration. This has set the stage to use CCMV capsids as enzyme nanoreactors at non-native and physiological conditions.

Lysozyme was chosen as the model enzyme to be encapsulated in the nanoreactor. This enzyme plays a key role in the human immune system, as it protects our body from Gram-positive pathogens, such as Bacillus and Streptococcus. It does so by cleaving the core $\beta$ - $(1,4)$ glycosidic bond between $\mathrm{N}$-acetylmuramic acid and the fourth carbon atom of the $\mathrm{N}$ acetylglucosamine of bacterial cell wall peptidoglycans, resulting in bacterial lysis and death. ${ }^{14}$ Human lysozyme has been successfully applied in early studies to treat experimentally induced pneumonia using hamster models, which has laid the foundation for clinical testing of lysozyme for antibiotic treatments. ${ }^{15,16}$ The enzyme has some disadvantageous properties, however, which hamper its application in a clinical setting. For instance, the cationic character of lysozyme can lead to aggregation with negatively charged biopolymers, resulting in inhibition of its antibacterial properties. Additionally, lysozymespecific inhibitors exist, which also limits the clinical efficacy of lysozyme. So far, only genetic engineering has been used to address these problems. ${ }^{17-20}$ In some cases, the engineered variants did indeed show improved properties. Changing the lysozyme genetic sequence, however, has also led to the introduction of new unexpected and unfavorable properties.

We set out to encapsulate lysozyme in a nanocage structure, in order to protect it against harmful external conditions or molecules, allowing the enzyme to remain active without the need for extensive genetic engineering of the T4L sequence. Since the lysozyme activity drops significantly upon an increase in ionic strength, a nanoreactor is required which is stable under physiological conditions. ${ }^{21,22}$ We hypothesized that the metal ion-stabilized ELP-CCMV particles might be very suitable nanocarriers for this purpose.

In order to encapsulate lysozyme, statistical encapsulation during assembly of the ELP-CCMV capsids would probably not yield a high enough encapsulation efficiency due to the cationic character of lysozyme. Therefore, we chose to use a covalent coupling strategy, which has previously been published by our group. ${ }^{23,24}$ Briefly, we used Sortase A (SrtA) to modify the Nterminus of ELP-CCMV with different cargoes, which eventually end up at the interior of the VLPs (Figure 1).
SrtA is present in Gram-positive bacteria, where it anchors surface proteins to the bacterial cell wall. It recognizes proteins with the sorting signal LPXTG (where $\mathrm{X}=$ any amino acid). SrtA cleaves the bond between threonine and glycine, after which an $\mathrm{N}$-terminal glycine residue attacks the intermediate.

We aimed to introduce the sorting signal LPETG onto the C-terminus of T4 lysozyme (T4L), using a single step of genetic engineering, in order to couple it to the $\mathrm{N}$-terminus of ELP-CCMV using SrtA (Figure 1). After optimization of the coupling reaction and subsequent assembly and metal ionmediated stabilization of the capsids, we investigated the activity of lysozyme after coupling to ELP-CCMV by monitoring the degradation of the cell walls of Micrococcus luteus (M. luteus), a Gram-positive bacterium.

\section{EXPERIMENTAL SECTION}

Materials. Hot start II HF DNA polymerase, dNTPs, restriction enzymes, and T4 DNA ligase were obtained from New England Biolabs. The DNA oligos were synthesized by Biolegio. Ampicillin was purchased from MP Biomedicals. Chloramphenicol was obtained from Sigma-Aldrich. Isopropyl $\beta$-D-1-thiogalactopyranoside (IPTG) was purchased from Acros. Ni-NTA agarose beads were obtained from Qiagen. The fluorescein conjugated Micrococcus luteus was purchased as part of the EnzChek Lysozyme Assay Kit from Fisher Scientific.

UV-Vis Absorbance Measurements. Protein concentrations were measured on a Varian Cary 50 Conc UV-vis spectrometer using a quartz cuvette with a path length of $3 \mathrm{~mm}$. Protein concentrations were calculated using the theoretical extinction coefficients. ${ }^{25}$ Samples were centrifuged prior to the measurements.

Mass Spectrometry. Protein mass characterization was performed by electrospray ionization time-of-flight (ESI-TOF) on a JEOL AccuTOF CS. Deconvoluted mass spectra were obtained using MagTran 1.03 b2. Isotopically averaged molecular weights were calculated using the "Protein Calculator v3.4" at http://protcalc. sourceforge.net. Protein samples were desalted by spin filtration with MQ (final concentrations approximately $20 \mu \mathrm{M}$ ).

Size Exclusion Chromatography (SEC). SEC measurements were performed on a Superose 6 increase 10/300 column (GE Healthcare). Analytical and preparative SEC measurements were executed on a Shimadzu LC-2010AHT HPLC and Agilent 1260 bioinert HPLC, respectively. Samples were separated on the column with a flow rate of $0.5 \mathrm{~mL} / \mathrm{min}$.

Transmission Electron Microscopy (TEM). TEM grids (FCF200-Cu, EMS) were glow-discharged using a Cressington carbon coater and power unit. Protein samples $(0.2 \mathrm{mg} / \mathrm{mL}, 5 \mu \mathrm{L})$ were applied on the glow-discharged grids and incubated for $1 \mathrm{~min}$. The samples were carefully removed using a filter paper and the grid was 
allowed to dry for at least $15 \mathrm{~min}$. Then the grid was negatively stained by applying $2 \%$ uranyl acetate in water $(5 \mu \mathrm{L})$. The staining solution was removed after $15 \mathrm{~s}$ and the grid was allowed to dry for at least 15 min. The samples were analyzed on a JEOL JEM-1010 TEM.

Microplate Reader Experiments. Microplate reader experiments were performed on a Tecan Spark $10 \mathrm{M}$ microplate reader at $21^{\circ} \mathrm{C}$. Samples were centrifuged prior to analysis. Buffers were filtered prior to use. Excitation and emission wavelengths were set to 485 and 530 $\mathrm{nm}$, respectively. All measurements were done in triplicate.

Dynamic Light Scattering (DLS) Measurements. DLS measurements were performed on a Malvern Zetasizer Nano ZSP at $20{ }^{\circ} \mathrm{C}$. Samples were centrifuged prior to analysis. Buffers were filtered prior to use. All measurements were done in triplicate, and the average of the triplicate measurements was plotted.

SrtA-Mediated Coupling Experiments. For a typical SrtAmediated coupling experiment, stock solutions of SrtA, G-ELPCCMV, and T4L-LPETG were prepared in Sortase buffer. If a component had been dissolved in another buffer, it was spin filtrated to Sortase buffer $(10 \mathrm{kDa}$ MWCO, $3 \times 10 \mathrm{~min})$. The components were added together to final concentrations of $0-100 \mu \mathrm{M}$ SrtA, $50 \mu \mathrm{M}$ G-ELP-CCMV, and $50 \mu \mathrm{M}$ T4L-LPETG. The solutions were shaken at $21{ }^{\circ} \mathrm{C}$ for $24 \mathrm{~h}$. The reaction progress was followed by SDS-PAGE analysis.

SrtA-Mediated Coupling Experiments, Followed by Capsid Purification. For a typical SrtA-mediated coupling experiment, stock solutions of SrtA, G-ELP-CCMV, and T4L-LPETG were prepared in Sortase buffer. If a component had been dissolved in another buffer, it was spin filtrated to Sortase buffer ( $10 \mathrm{kDa}$ MWCO, $3 \times 10 \mathrm{~min})$. The components were added together to final concentrations of $0 / 10 \mu \mathrm{M}$ SrtA, $50 \mu \mathrm{M}$ G-ELP-CCMV, and 0/50 $\mu \mathrm{M}$ T4L-LPETG. The solutions were shaken at $21{ }^{\circ} \mathrm{C}$ for $3 \mathrm{~h}$. The capsids were assembled to either pH 5.0 capsid buffer by spin filtration (10 kDa MWCO, $3 \times$ $10 \mathrm{~min}$ ) or $\mathrm{pH} 7.5$ capsid buffer by dialysis (Spectra/Por 2 dialysis tubing, $12-14 \mathrm{kDa}$ MWCO, $25 \mathrm{~mm}$ flat width, $3 \times 20 \mathrm{~min}$ ). Subsequently, the capsids were isolated using preparative SEC. The combined capsid fractions were concentrated by spin filtration, analyzed, and used for further experiments.

Activity Assay with Free T4L. Lysozyme activity of free T4L and T4L-LPETG was measured by observing the degradation of fluorescently labeled $M$. luteus cell walls (EnzChek lysozyme assay). An increase in fluorescence indicated the presence of active lysozyme, as the degradation of the cell walls reduced the quenching of the fluorescent groups. The fluorescently labeled substrate was diluted from $1 \mathrm{mg} / \mathrm{mL}$ in $\mathrm{MQ}$ to $50 \mu \mathrm{M}$ in the desired buffer. The substrate $(50 \mu \mathrm{L})$ was added to T4L or T4L-LPETG $(50 \mu \mathrm{L}, 2 \mu \mathrm{M}$ in the desired buffer) in triplicate in a 96-well plate. The fluorescence intensity was then followed for $60 \mathrm{~min}$ at $21{ }^{\circ} \mathrm{C}$ with intermediate shaking steps on a Tecan Spark $10 \mathrm{M}$ microplate reader (excitation wavelength $485 \mathrm{~nm}$, emission wavelength $530 \mathrm{~nm}$ ). The initial slopes of the curves were taken as a measure of the hydrolytic activity.

Activity Assay with Encapsulated T4L (and Related Controls). The isolated and concentrated capsid fractions (see SrtAMediated Coupling Experiments, Followed by Capsid Purification) were diluted to a concentration of approximately $200 \mu \mathrm{M}$ by measuring the $\mathrm{OD}_{280}$ of the concentrated capsid solution and diluting to an $\mathrm{OD}_{280}$ of 1.4. Subsequently, $\mathrm{NiCl}_{2}(900 \mu \mathrm{M}$ in either $\mathrm{pH} 5.0$ capsid buffer or $\mathrm{pH} 7.5$ capsid buffer) or buffer (equal volume as the capsid solution) was added (final protein concentration is approximately $100 \mu \mathrm{M}$ ). The mixtures were incubated for $30 \mathrm{~min}$ at room temperature. Subsequently, the mixtures were dialyzed to reaction buffer (Spectra/Por 4 dialysis tubing, 12-14 kDa MWCO, $10 \mathrm{~mm}$ flat width, $3 \times 20 \mathrm{~min}$; solutions with $\mathrm{Ni}^{2+}$ separately from solutions without $\mathrm{Ni}^{2+}$ ions, to prevent potential capsid formation in the control samples during dialysis). The activity assay with the capsids was then executed as described in the section Activity Assay with Free T4L, using $50 \mu \mathrm{L}$ of the capsid solution of approximately $100 \mu \mathrm{M}$ per reaction.

\section{RESULTS AND DISCUSSION}

The ELP-CCMV construct was designed such, that it displayed a glycine residue on its N-terminus for the SrtA reaction (GELP-CCMV). A T4L construct was modified to display the Sortase recognition motif LPETG at its C-terminus (T4LLPETG). A hexahistidine tag was additionally included at the C-terminus of T4L-LPETG, for facile purification via affinity chromatography. As a control, a T4L construct was prepared with a C-terminal hexahistidine tag, but lacking the LPETG recognition motif. The proteins were subsequently expressed in Escherichia coli (E. coli), followed by purification through $\mathrm{Ni}^{2+}$ affinity chromatography (see Supporting Information for experimental procedures and characterization of the expressed proteins).

Next, the Sortase-mediated coupling of T4L-LPETG to GELP-CCMV was evaluated. To this end, different equivalents of SrtA were added to a mixture of T4L-LPETG and G-ELPCCMV. The coupling reaction was followed over time by polyacrylamide gel electrophoresis (SDS-PAGE; Figure 2A). Formation of both the intermediate T4L-SrtA and the desired product T4L-ELP-CCMV could be observed over time. As

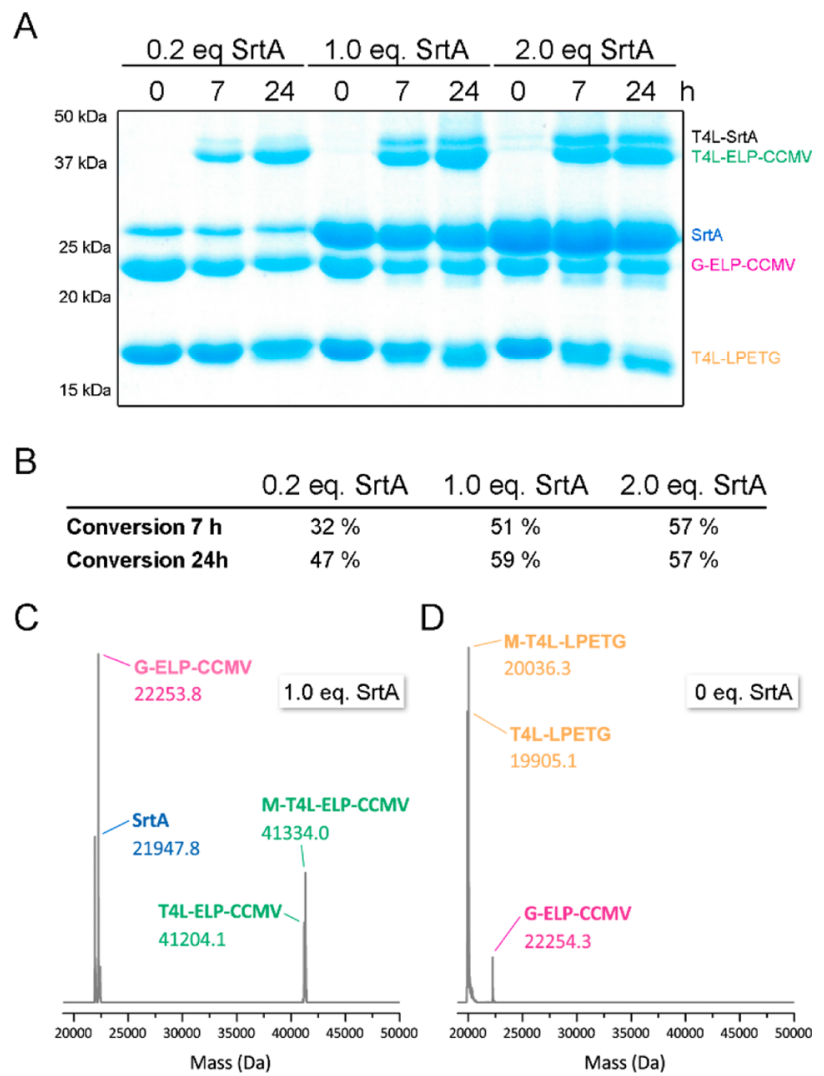

Figure 2. Analysis of the SrtA-mediated coupling of T4L-LPETG to G-ELP-CCMV. (A) SDS-PAGE analysis of the conjugation over time, with different equivalents of SrtA. Protein bands were visualized by Coomassie blue staining. (B) Conversions of G-ELP-CCMV to T4LLPETG-ELP-CCMV after 7 and $24 \mathrm{~h}$, based on quantitative Image analysis of the SDS-PAGE results shown in (A). (C) Deconvoluted ESI-TOF mass spectrum of a reaction mixture with 1.0 equiv SrtA after $24 \mathrm{~h}$. (D) Deconvoluted ESI-TOF mass spectrum of a reaction mixture with 0 equiv SrtA after $24 \mathrm{~h}$. The expected molecular weights are 21947.5 Da (SrtA, blue), 22253.4 Da (G-ELP-CCMV, pink), 19904.6/20035.8 Da (T4L-LPETG/M-T4L-LPETG, yellow), and 41203.1/41334.3 Da (T4L-ELP-CCMV/M-T4L-ELP-CCMV, green). 
expected, the addition of higher equivalents of SrtA, compared to the reactants, resulted in a faster reaction and more product formation after $24 \mathrm{~h}$, as indicated by quantitative Image analysis of the SDS-PAGE results (Figure 2B). Product formation was further confirmed by electrospray ionization time-of-flight (ESI-TOF) mass spectrometry analysis of the reaction mixtures after $24 \mathrm{~h}$ (Figure $2 \mathrm{C}$ ). No product formation was observed using SDS-PAGE and ESI-TOF analysis when no SrtA was added to the reaction mixture (Figures S1 and 2D).

A conventional way to monitor lysozyme activity is to follow degradation of lyophilized $M$. luteus cells. In this assay, a decrease in turbidity of the cell wall suspension indicates the presence of active lysozyme. Even though this assay has been the benchmark in lysozyme activity assays for a long time, the sensitivity and reproducibility of this assay are not optimal. In another procedure, the $M$. luteus cell walls are fluorescently labeled to such a degree, that the fluorescence becomes quenched. If now lysozyme-catalyzed degradation of the cell walls takes place, a dramatic increase of fluorescence occurs, making this assay much more sensitive. We chose to use the fluorescence-based assay for the next series of experiments.

First, the activity of hexahistidine-tagged T4L and the LPETG-modified variant were tested in the benchmark phosphate buffer $\mathrm{pH}$ 7.5, supplied with the substrate kit (Figure S2). Significant activity was observed for both enzymes under the experimental conditions used. A slight decrease in activity was observed as a consequence of the introduction of the LPETG sequence in T4L-LPETG. Next, we tested whether T4L would become inactive under conditions at which the GELP-CCMV capsid proteins are assembled, that is, $\mathrm{pH} 5.0$ capsid buffer or $\mathrm{pH} 7.5$ capsid buffer with $2.0 \mathrm{M} \mathrm{NaCl}$. In neither buffer, activity was observed. Also, in $\mathrm{pH} 7.5$ dimer buffer, containing $500 \mathrm{mM} \mathrm{NaCl}$, no activity could be observed. Reducing the $\mathrm{NaCl}$ concentration of the $\mathrm{pH} 7.5$ dimer buffer to $150 \mathrm{mM}$ did result in lysozyme activity. Additionally, lowering the Tris $\mathrm{HCl}$ concentration from 50 to $10 \mathrm{mM}$ further increased the activity. At $\mathrm{pH} 5$, no activity was observed at all, even upon lowering the ionic strength of the buffer. These results confirm that, in order to ensure activity of the encapsulated T4L, further stabilization of the G-ELP-CCMV capsids with metal ions is required as no lysozyme activity was observed in buffers in which the capsids remain assembled without additional stabilization.

Subsequently, a protocol was developed for capsid assembly and stabilization from a SrtA reaction mixture, containing modified and nonmodified G-ELP-CCMV capsid protein dimers, SrtA and free T4L-LPETG. This was done based on previous work on both the SrtA-mediated modification of the $\mathrm{N}$-terminus of CCMV capsid proteins and $\mathrm{Ni}^{2+}$-induced capsid formation of ELP-CCMV. ${ }^{13,23,24}$ In the first attempt, $\mathrm{Ni}^{2+}$ ions were directly added to the reaction mixture to induce the formation of stabilized capsids, followed by preparative size exclusion chromatography (SEC) purification of the assembled capsids. This, however, did not lead to the formation of monodisperse capsids, as indicated by SEC analysis (Figure S3). Instead, tailing of the capsid peak was observed and a very low amount of capsids was obtained. A possible explanation could be that the $\mathrm{Ni}^{2+}$ ions also interact with other proteins in the solution with a hexahistidine tag, such as SrtA and free T4LLPETG. Therefore, it was decided to first induce capsid formation from the SrtA reaction mixture via either $\mathrm{pH}$-induced assembly or salt-induced assembly, followed by preparative SEC purification and subsequent stabilization of the capsids with
$\mathrm{Ni}^{2+}$ ions. Both assembly methods, that is, lowering the $\mathrm{pH}$ of the reaction mixture from 7.5 to 5.0 or increasing the $\mathrm{NaCl}$ concentration of the reaction mixture from 150 to $2000 \mathrm{mM}$, were tested, and both resulted in the formation of capsids from the reaction mixtures (Figure 3A). After successful purification
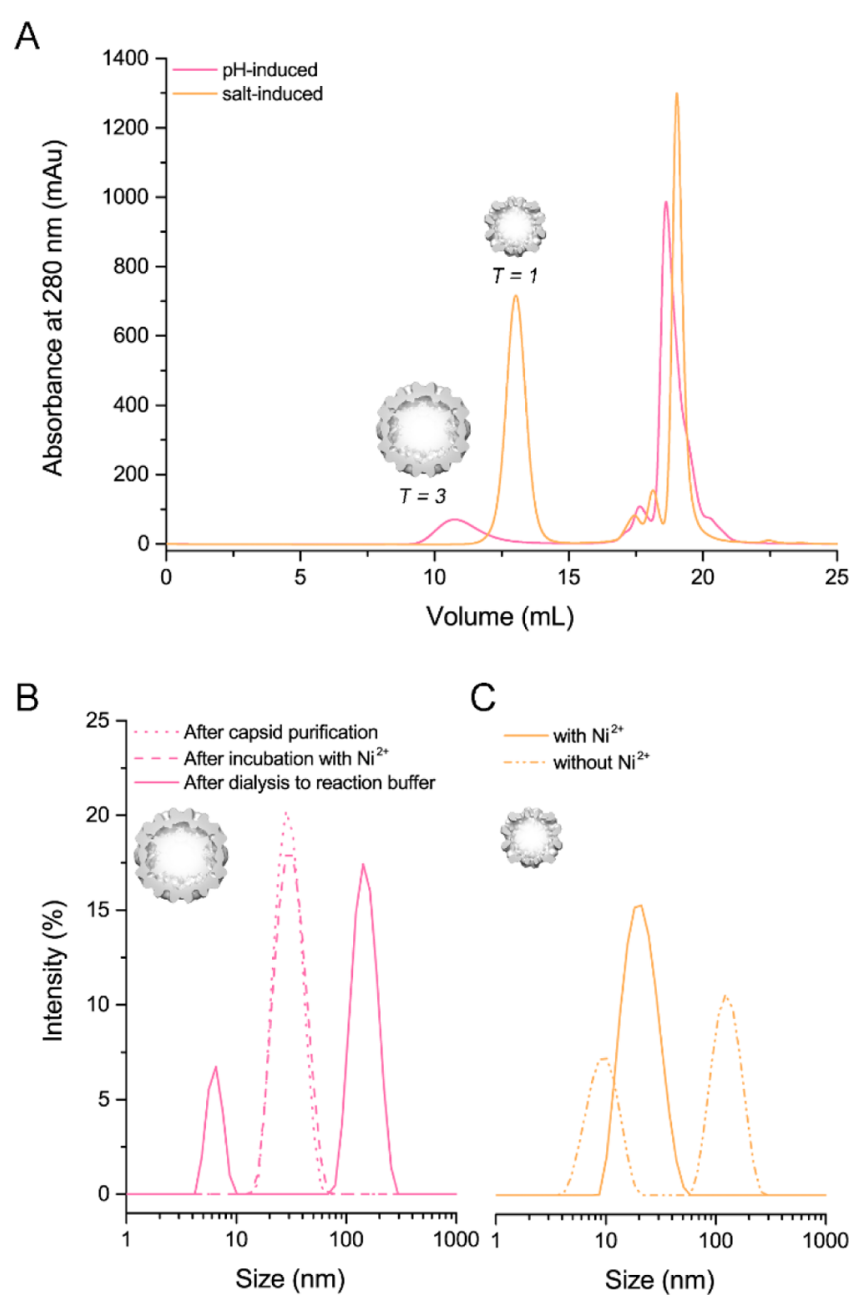

Figure 3. (A) Size exclusion chromatograms of SrtA reaction mixtures after dialysis to either pH 5.0 capsid buffer (pH-induced, pink line) or $\mathrm{pH} 7.5$ capsid buffer containing $2.0 \mathrm{M} \mathrm{NaCl}$ (salt-induced, orange line). Capsids are observed around an elution volume of $10-13 \mathrm{~mL}$, with the $T=3$ particles eluting earlier due to their larger size. (B) DLS measurements of the $T=3$ capsid fraction shown in (A) after preparative SEC purification, after subsequent incubation with $\mathrm{Ni}^{2+}$ ions, and after subsequent dialysis to reaction buffer. (C) DLS measurements of the $T=1$ capsid fraction shown in (A), after incubation with (solid line) or without (dashed line) $\mathrm{Ni}^{2+}$ ions, followed by dialysis to reaction buffer. Capsids are expected around 20-30 nm, and dimers are expected below $10 \mathrm{~nm}$.

of both the $T=3$ and $T=1$ capsids, it was tested whether these capsids could be stabilized by $\mathrm{Ni}^{2+}$ ions. To this end, the capsids were incubated with $\mathrm{Ni}^{2+}$ ions and subsequently dialyzed to the T4L-compatible $\mathrm{pH} 7.5$ dimer buffer with reduced $\mathrm{NaCl}$ and Tris $\cdot \mathrm{HCl}$ concentrations (will be referred to as reaction buffer). DLS analysis showed that the $T=3$ capsids fell apart during dialysis, indicating that the stabilizing effect of the metal ions is not enough in this case (Figure $3 \mathrm{~B}$ ). The $T=1$ particles, however, could be stabilized by $\mathrm{Ni}^{2+}$ : capsids were still present after dialysis to the reaction buffer (Figure 3C). Transmission electron microscopy (TEM) analysis of the particles directly 
after preparative SEC in $\mathrm{pH} 7.5$ capsid buffer, and after stabilization and dialysis to reaction buffer showed equally sized particles of approximately $19.6 \mathrm{~nm}$ (Figure S4). When the $\mathrm{Ni}^{2+}$ ions were left out, disassembly toward the capsid protein dimers occurred during dialysis, as expected. We therefore continued with the stabilized $T=1$ particles for the remaining experiments.

After having developed a successful protocol for the formation and stabilization of G-ELP-CCMV $T=1$ capsids after the coupling reaction with T4L-LPETG, the lysozyme encapsulation efficiency was determined. To this end, the composition of the isolated capsid fraction was analyzed by SDS-PAGE (Figure 4A). As a control, capsids were prepared

A

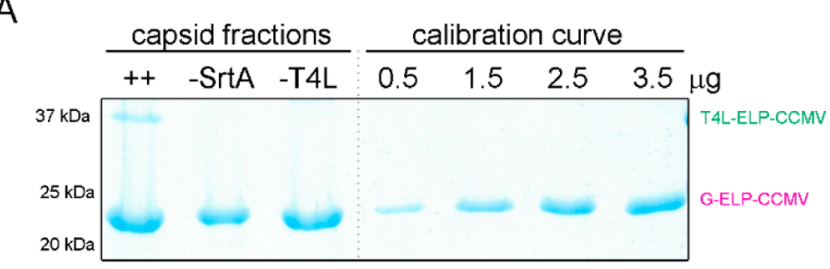

B

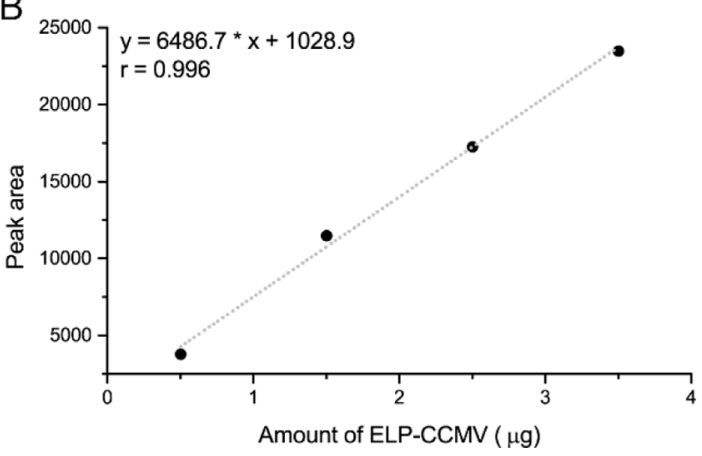

Figure 4. SDS-PAGE analysis of isolated capsid fractions after the SrtA-mediated coupling of T4L-LPETG to G-ELP-CCMV. (A) Left: comparison between capsids prepared from reaction mixtures containing both SrtA and T4L-LPETG $(++)$, no SrtA (-SrtA), and no T4L-LPETG (-T4L). Right: calibration curve of G-ELP-CCMV. Protein bands were visualized by Coomassie blue staining. (B) Calibration curve of the peak area observed on SDS-PAGE, related to the amount of G-ELP-CCMV in $\mu \mathrm{g}$. The calibration curve was constructed based on the SDS-PAGE results shown in (A).

from reaction mixtures lacking either SrtA or T4L-LPETG. A band corresponding to G-ELP-CCMV was observed for all three capsid samples. As expected, the coupled product T4LELP-CCMV was only observed when both SrtA and T4LLPETG had been present during the coupling reaction. ImageJ analysis of this gel suggested that each $T=1$ capsid contained approximately $4 \mathrm{~T} 4 \mathrm{~L}$ enzymes. This is quite a remarkable amount of enzymes to fit in such a small compartment, taking into account that lysozyme has a cationic surface charge.

To investigate the enzymatic activity of T4L after encapsulation in the capsids, the capsids in the reaction buffer were exposed to $M$. luteus cell walls, labeled with fluorophores of which the fluorescence was self-quenched (Figure 5). Also dimers, obtained from the procedure where the $\mathrm{Ni}^{2+}$ ions had been left out before dialysis to reaction buffer, were added to the M. luteus substrate. As a control, samples were prepared from SrtA reaction mixtures deficient of either SrtA or T4LLPETG. The T4L-LPETG concentration in the active capsid

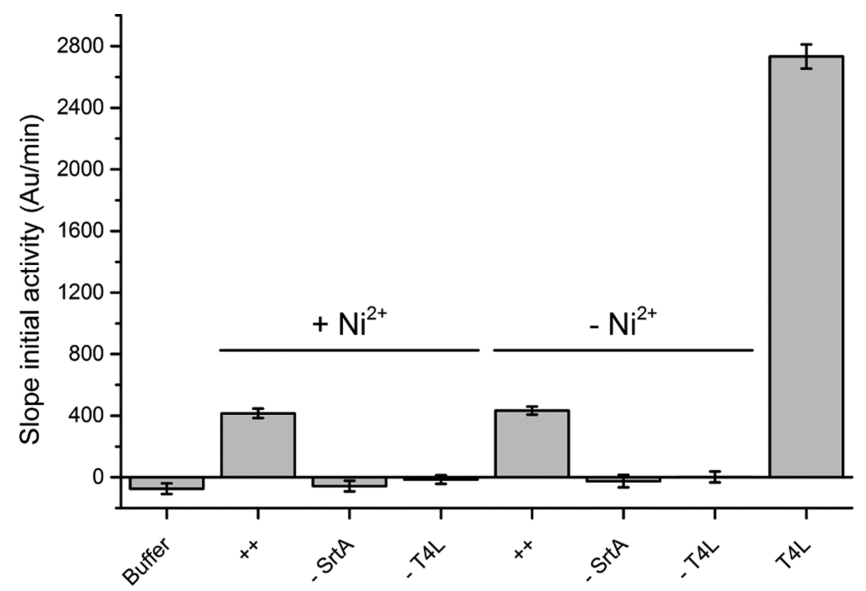

Figure 5. Catalytic activity of capsids where both SrtA and T4LLPETG had been present during the SrtA reaction $(++)$ or where either SrtA or T4L-LPETG had been left out (-SrtA and -T4LLPETG, respectively). The activity was measured using the EnzChek lysozyme assay. $+\mathrm{Ni}^{2+}$ : nickel ions were added before dialysis to reaction buffer, to stabilize the capsids; $-\mathrm{Ni}^{2+}$ : nickel ions were not added before dialysis to reaction buffer, yielding dimers. The activity of the ++ samples was compared to the activity of nonencapsulated T4LLPETG, in approximately the same concentration as the capsid samples. As a control, the background reaction in reaction buffer was measured.

and dimer samples was determined based on the ratio of T4LELP-CCMV and G-ELP-CCMV, determined by SDS-PAGE analysis (Figure 4A) and comparison to a calibration curve of G-ELP-CCMV (Figure 4B). Only when both SrtA and T4LLPETG had been present during the reaction, a fluorescence increase was observed. This showed that the cargo was available to react with the substrate after encapsulation, as shown previously when CalB was encapsulated using a similar strategy. $^{24}$ Free T4L-LPETG, in the same concentration as present in the active capsid and dimer samples, was more active (i.e., approximately seven times) than T4L-LPETG attached to G-ELP-CCMV (Figure 5). Additionally, free T4L-LPETG, subjected to the same protocol, but without SrtA and G-ELPCCMV present, was equally active as T4L-LPETG that had not been subjected to this protocol (Figure S5). Hence, attachment of ELP-CCMV did affect lysozyme activity to some extent. Attempts to disassemble the metal ion-stabilized capsids into capsids by the addition of an excess of chelating agent ethylenediaminetetraacetic acid (EDTA) failed. Probably, the crowded interior of the loaded capsids did not allow EDTA to reach the metal ions near the hexahistidine tags.

Surprisingly, the activities of the T4L-modified dimers and capsids were very similar (Figure 5). Even if the M. luteus cells had been fragmented during lyophilization, resulting in cell wall fragments which could more easily come into contact with enzyme molecules, we would still expect to see a difference in activity, that is, some effect of diffusion through the pores of the $T=1$ capsids. To be sure that the addition of the substrate did not result in capsid disassembly, DLS measurements were performed on the capsids before and after substrate addition (Figure S6). No signs of capsid disassembly were observed, suggesting that the capsids remained intact during the activity assay. We could also rule out aspecific adhesion of free T4LLPETG to the outside of the capsids, as no free T4L-LPETG was observed on the SDS-PAGE gels after capsid purification (Figure 4A). Lastly, the effect of G-ELP-CCMV itself on T4L 
activity was investigated by incubating free T4L-LPETG with free G-ELP-CCMV, followed by a measurement of the lysozyme activity (Figure S7). No effect of the presence of G-ELP-CCMV was observed on the T4L activity. This excludes any effects arising from aspecific ionic interactions between lysozyme and the CCMV capsid protein. From the experiments above, we can conclude that the T4L enzyme molecules retain their activity after encapsulation inside the CCMV capsids and that their activity is somewhat reduced compared to free T4L.

\section{CONCLUSION}

We have utilized a protein-based block copolymer of CCMV and its expanded assembly profile to create nanoreactors which can function under conditions with similar salt concentrations as present in the physiological environment. As a proof-ofprinciple, T4 lysozyme was successfully encapsulated via selective attachment to the interior of the CCMV capsids. The activity of this enzyme is highly dependent on the salt concentration and $\mathrm{pH}$ of the environment. Only after addition of metal ions to the CCMV capsids, these could remain stable at conditions at which its cargo was also active. A high encapsulation efficiency of approximately 4 enzymes per $T=1$ capsid was achieved, indicating that the unfavorable interaction between the cationic lysozyme molecules was overcome. Possibly these charges were neutralized by interactions with the interior of the capsids. It was shown that on incorporation in the CCMV capsids, the enzyme molecules were available to interact with the substrate and remained active. This opens perspectives to use the shielded T4L in antibiotic applications. Attempts to disassemble the capsids by the addition of EDTA, which should bind the metal ions, have not been successful so far. Future research could focus on methods to disassemble these capsids, and expansion of the assembly/disassembly cycle toward a switchable nanoreactor. Furthermore, we believe this strategy is widely applicable to different types of cargo, and provides interesting opportunities for the in vitro and possible in vivo application of the CCMV capsids.

\section{ASSOCIATED CONTENT}

\section{S Supporting Information}

The Supporting Information is available free of charge on the ACS Publications website at DOI: 10.1021/acs.biomac.7b00640.

Cloning and expression of proteins and supplemental figures (PDF).

\section{AUTHOR INFORMATION}

\section{Corresponding Author}

*Tel.: +3140-2473515. E-mail: j.c.m.v.hest@tue.nl.

\section{ORCID}

Jan C. M. van Hest: 0000-0001-7973-2404

\section{Author Contributions}

The manuscript was written through contributions of all authors. All authors have given approval to the final version of the manuscript.

\section{Notes}

The authors declare no competing financial interest.

\section{ACKNOWLEDGMENTS}

The authors thank Linda J. A. Hendriks for her initial involvement in the project. The Ministry of Education, Culture and Science (Gravitation Program 024.001.035) is acknowledged for financial support.

\section{ABBREVIATIONS}

CCMV, cowpea chlorotic mottle virus; ELP, elastin-like polypeptide; ESI-TOF, electrospray ionization time-of-flight; E. coli, Escherichia coli; EDTA, ethylenediaminetetraacetic acid; M. luteus, Micrococcus luteus; SDS-PAGE, sodium dodecyl sulfate polyacrylamide gel electrophoresis; SEC, size exclusion chromatography; SrtA, sortase A; T4L, T4 lysozyme; VLPs, virus-like particles

\section{REFERENCES}

(1) Douglas, T.; Young, M. Science 2006, 312, 873-875.

(2) Caspar, D. L. D.; Klug, A. Cold Spring Harbor Symp. Quant. Biol. 1962, 27, 1-24.

(3) Speir, J. A.; Munshi, S.; Wang, G.; Baker, T. S.; Johnson, J. E. Structure 1995, 3, 63-78.

(4) Lavelle, L.; Gingery, M.; Phillips, M.; Gelbart, W. M.; Knobler, C. M.; Cadena-Nava, R. D.; Vega-Acosta, J. R.; Pinedo-Torres, L. A.; Ruiz-Garcia, J. J. Phys. Chem. B 2009, 113, 3813-3819.

(5) Douglas, T.; Young, M. Nature 1998, 393, 152-155.

(6) Liepold, L.; Anderson, S.; Willits, D.; Oltrogge, L.; Frank, J. A.; Douglas, T.; Young, M. Magn. Reson. Med. 2007, 58, 871-879.

(7) Sikkema, F. D.; Comellas-Aragonès, M.; Fokkink, R. G.; Verduin, B. J. M.; Cornelissen, J. J. L. M.; Nolte, R. J. M. Org. Biomol. Chem. 2007, 5, 54-57.

(8) Minten, I. J.; Ma, Y.; Hempenius, M. A.; Vancso, G. J.; Nolte, R. J. M.; Cornelissen, J. J. L. M. Org. Biomol. Chem. 2009, 7, 4685-4688.

(9) Comellas-Aragonès, M.; Engelkamp, H.; Claessen, V. I.; Sommerdijk, N. A. J. M.; Rowan, A. E.; Christianen, P. C. M.; Maan, J. C.; Verduin, B. J. M.; Cornelissen, J. J. L. M.; Nolte, R. J. M. Nat. Nanotechnol. 2007, 2, 635-639.

(10) van Eldijk, M. B.; Wang, J. C.-Y.; Minten, I. J.; Li, C.; Zlotnick, A.; Nolte, R. J. M.; Cornelissen, J. J. L. M.; van Hest, J. C. M. J. Am. Chem. Soc. 2012, 134, 18506-18509.

(11) Urry, D. W. J. Phys. Chem. B 1997, 101, 11007-11028.

(12) van Eldijk, M. B.; McGann, C. L.; Kiick, K. L.; van Hest, J. C. M. Top. Curr. Chem. 2011, 310, 71-116.

(13) van Eldijk, M. B.; Schoonen, L.; Cornelissen, J. J. L. M.; Nolte, R. J. M.; van Hest, J. C. M. Small 2016, 12 (18), 2476-2483.

(14) Callewaert, L.; Michiels, C. W. J. Biosci. 2010, 35, 127-160.

(15) Bhavsar, T.; Liu, M.; Hardej, D.; Liu, X.; Cantor, J. Exp. Lung Res. 2010, 36, 94-100.

(16) Bhavsar, T.; Liu, M.; Liu, X.; Cantor, J. Exp. Lung Res. 2011, 37, $536-541$.

(17) Gill, A.; Scanlon, T. C.; Osipovitch, D. C.; Madden, D. R.; Griswold, K. E. PLoS One 2011, 6, e16788.

(18) Teneback, C. C.; Scanlon, T. C.; Wargo, M. J.; Bement, J. L.; Griswold, K. E.; Leclair, L. W. Antimicrob. Agents Chemother. 2013, 57, $5559-5564$.

(19) Griswold, K. E.; Bement, J. L.; Teneback, C. C.; Scanlon, T. C.; Wargo, M. J.; Leclair, L. W. Bioengineered 2014, 5, 143-147.

(20) Dostal, S. M.; Fang, Y.; Guerrette, J. C.; Scanlon, T. C.; Griswold, K. E. ACS Chem. Biol. 2015, 10, 1110-1117.

(21) Davies, R. C.; Neuberger, A.; Wilson, B. M. Biochim. Biophys. Acta 1969, 178, 294-305.

(22) Jensen, H. B.; Kleppe, K. Eur. J. Biochem. 1972, 28, 116-122.

(23) Schoonen, L.; Pille, J.; Borrmann, A.; Nolte, R. J. M.; van Hest, J. C. M. Bioconjugate Chem. 2015, 26 (12), 2429-2434.

(24) Schoonen, L.; Nolte, R. J. M.; van Hest, J. C. M. Nanoscale 2016, 8, 14467-14472.

(25) Gill, S. C.; von Hippel, P. H. Anal. Biochem. 1989, 182, 319326. 